\title{
Physiological and Molecular Characterization of a Synechocystis sp. PCC 6803 Mutant Lacking Histidine Kinase Slr1759 and Response Regulator SIr1760
}

Anke Nodop ${ }^{\mathrm{a}}$, Iwane Suzuki ${ }^{\mathrm{b}, \mathrm{d}}$, Aiko Barsch ${ }^{\mathrm{c}}$, Ann-Kristin Schröder ${ }^{\mathrm{a}}$, Karsten Niehaus ${ }^{c}$, Dorothee Staiger ${ }^{a}$, Elfriede K. Pistorius ${ }^{a}$, and Klaus-Peter Michel ${ }^{\mathrm{a}, *}$

a Universität Bielefeld, Biologie VIII: Molekulare Zellphysiologie, D-33501 Bielefeld, Germany. E-mail: klauspeter.michel@uni-bielefeld.de

b Division of Cellular Regulation, National Institute for Basic Biology, Myodaiji, Okazaki 444-8585, Japan

c Universität Bielefeld, Biologie VI: Genetik, D-33501 Bielefeld, Germany

d Present address: Graduate School of Life and Environmental Sciences, University of Tsukuba, Tsukuba, Ibaraki 305-8572, Japan

* Author for correspondence and reprint requests

Z. Naturforsch. 61c, 865-878 (2006); received June 6/30, 2006

The hybrid sensory histidine kinase Slr1759 of the cyanobacterium Synechocystis sp. strain PCC 6803 contains multiple sensory domains and a multi-step phosphorelay system. Immuno blot analysis provided evidence that the histidine kinase Slr1759 is associated with the cytoplasmic membrane. The gene slr1759 is part of an operon together with slr1760, encoding a response regulator. A comparative investigation was performed on Synechocystis sp. strain PCC 6803 wild type (WT) and an insertionally inactivated slr1759-mutant (Hik14) which also lacks the transcript for the response regulator Slr1760. The mutant Hik14 grew significantly slower than WT in the early growth phase, when both were inoculated with a low cell density into BG11 medium without additional buffer and when aerated with air enriched with $2 \%$ $\mathrm{CO}_{2}$. Since the aeration with $\mathrm{CO}_{2}$-enriched air results in a decrease of the $\mathrm{pH}$ value in the medium, the growth experiments indicated that Hik14 is not able to adjust its metabolic activities as rapidly as WT to compensate for a larger decrease of the $\mathrm{pH}$ value in the medium. No significant differences in growth between Hik14 and WT were observed when cells were inoculated with a higher cell density in BG11 medium or when the BG11 medium contained $50 \mathrm{~mm}$ Epps-NaOH, pH 7.5, to prevent the $\mathrm{pH}$ drop. This Hik14 phenotype has so far only been seen under the above defined growth condition. Results of photosynthetic activity measurements as well as Northern blot-, immuno blot-, and metabolite analyses suggest that the two-component system Slr1759/Slr1760 has a function in the coordination of several metabolic activities which is in good agreement with the complex domain structure of Slr1759. The direct targets of this two-component system have so far not been identified.

Key words: Synechocystis sp. PCC 6803, Two-Component System, Histidine Kinase 